\title{
Can the AGE/RAGE/ERK signalling pathway and the epithelial-to-mesenchymal transition interact in the pathogenesis of chronic rhinosinusitis with nasal polyps?
}

\author{
Antonella Vetuschi, ${ }^{1}$ Simona Pompili, ${ }^{1}$ Gian Piero Di Marco, ${ }^{2}$ Federico Calvaruso, ${ }^{1}$ Enzo Iacomino, ${ }^{2}$ \\ Lidia Angelosante, ${ }^{3}$ Claudio Festuccia, ${ }^{1}$ Alessandro Colapietro, ${ }^{1}$ Roberta Sferra ${ }^{1}$ \\ ${ }^{1}$ Department of Biotechnological and Applied Clinical Sciences, University of L'Aquila \\ ${ }^{2}$ ENT Division, San Salvatore Hospital, L'Aquila \\ ${ }^{3}$ Unit of Intensive Care, Department of Anaesthesia, San Salvatore Hospital, L'Aquila, Italy
}

Chronic rhinosinusitis with nasal polyps (CRSwNP) is a persistent sinonasal mucosa inflammatory disease with still unclear pathophysiologic mechanisms that imply events of tissue repair and structural remodelling. Several cascades seem to have a considerable role in the onset and progression of mucosa hyperproliferation in nasal polyps including transforming growth factor $\beta /$ Small mother against decapentaplegic (TGF $\beta /$ Smads), mitogenactivated protein kinases (MAPKs), advanced glycosylation end-products (AGEs) together with epithelial-tomesenchymal transition (EMT). Since many inflammatory mediators are reported to play important roles in the development of nasal polyps (NP) disease, this study aimed to analyse the correlation between the AGEs/receptor of advanced glycosylation end-products (RAGE)/extracellular signal-regulated kinase (ERK) signalling pathway and the main markers of EMT to better understand the influence that they exert on the remodelling of nasal mucous membranes in patients affected by CRSwNP vs normal controls. A total of 30 patients were enrolled in this study. Immunohistochemical analysis, using AGE, RAGE, p-ERK, MMP-3, TGF- $\beta 1$, Smad2/3, Collagen I-III, $\alpha$-SMA, E-cadherin, IL-6 and Vimentin antibodies, was performed. AGE, RAGE, ERK, p-ERK and MMP3 were also evaluated using western blot analysis. We observed an overexpression of the AGE/RAGE/p-ERK and the main mesenchymal markers of EMT (Vimentin and IL-6) in CRSwNP vs controls whereas the TGF- $\beta / S m a d 3$ pathway did not show any significant differences between the two groups of patients. These observations suggest a complex network of processes in the pathogenesis of NP, and the AGE/RAGE/ERK pathway and EMT might work together in promoting tissue remodelling in the formation of CRSwNP.

Key words: Chronic rhinosinusitis; nasal polyps; AGE/RAGE/ERK; epithelial-to-mesenchymal transition.

Correspondence: Roberta Sferra, Department of Biotechnological and Applied Clinical Sciences, Human Anatomy, University of L'Aquila, Via Vetoio, Coppito 2, 67100 L'Aquila, Italy. Tel. +39.0862.433574/433504; Fax+39.0862.433523. E-mail: roberta.sferra@univaq.it

Contributions: AV, study design, coordination and manuscript drafting; SP, FC, performing IHC and quantitative analyses; AC, CF performing Western blot analyses; GDM, LA, EI, provided the surgical specimens; RS, study supervision, critical manuscript revision. All the authors have read and approved the final version of the manuscript and agreed to be accountable for all aspects of the work.

Conflict of interest: The authors declare that they have no competing interests, and all authors confirm accuracy.

Availability of data and materials: All data generated or analyzed during this study are included in this published article.

Ethics approval: The Institutional Ethic Committee approved the investigation protocol (n. 9993).

Informed consent: Written informed consent was obtained.

Funding: This work was supported by the Fondazione Carispaq L'Aquila (grant number U1428.2018. UOAI.1291). 


\section{Introduction}

Chronic rhinosinusitis with nasal polyps (CRSwNP) is a chronic inflammatory condition of the upper respiratory tract. This inflammatory disease of the mucous membranes in the nasal and paranasal sinuses is characterised by persistent eosinophilic inflammation, thickened sinonasal secretion, stromal oedema and grape-like neoformations, namely nasal polyps (NP), located in the upper nasal cavities. CRSwNP shows a persistent course and a frequent recurrence with a considerably impact on patients' quality of life despite surgery and postoperative medical treatment and because of the poor therapeutic response, patients clinically report nasal obstruction, anterior/posterior rhinorrhoea, facial pain or facial pressure and impairment or reduction of smell, cough and fatigue. $^{1,2}$

Numerous host and environmental factors including anatomic disorders, genetic factors, viral, bacterial and fungal colonisation or infections, allergic inflammation, non-allergic inhalants such as occupational chemicals and dusts, and cigarette smoke play a role in initiating and perpetuating the mucosal inflammation in the sinonasal cavities. ${ }^{1,3-5}$

Well established histological features characterise the aspect of the nasal/paranasal wall during CRSwNP such as basal membrane thickness, glandular density alteration or their morphological impairment, persistent mucosal inflammation, vessel proliferation and increase in vascular permeability, abnormal deposition of extracellular matrix (ECM) with local oedema and ECM degradation, remodelling of the tunica mucosa and submucosa finally leading to polyp formation. ${ }^{6}$

As it occurs in other organs, ${ }^{7-9}$ during the dynamic process culminating in a structural reorganisation of the nasal/paranasal mucosa, fibroblasts are thought to be the crucial cytotype of ECM remodelling. Indeed, fibroblasts are responsible for not only the local recruitment of inflammatory cells (owing to their ability to influence the production of a variety of cytokines), ${ }^{10,11}$ but also to represent a source of intracellular molecules capable of initiating and amplifying inflammation and the subsequent collagen deposition. ${ }^{12}$

Transforming growth factor $\beta$ (TGF $\beta 1$ ) has been shown to be closely associated with chronic upper respiratory tract inflammatory diseases playing a fundamental role in the remodelling process through promoting transdifferentiation of fibroblasts into myofibroblast capable of synthesising ECM proteins. ${ }^{13}$ TGF $\beta 1$ expression is different in chronic rhinosinusitis without nasal polyps (CRSsNP) patients showing high levels of this growth factor respect to CRSwNP patients demonstrating a predominant eosinophilic inflammation with high levels of IL-5 and local IgE with low immunopositivity of TGF $\beta 1 .{ }^{13,14}$ The low TGF $\beta 1$ protein concentration in CRSwNP patients is also associated with a low degree of TGF $\beta 1 \mathrm{R}, \mathrm{p}$-Smads and collagen, indicating that the TGF $\beta 1 /$ Smads pathway is not responsible for inducing fibroblasts to synthesise ECM proteins. Since the TGF $\beta 1 /$ Smads pathway is not active in the upper airway remodelling process with formation of recurrent NP, this event is regulated by other distinct cascades. It is well known that in many organs tissue remodelling depends on the dynamic balance between enzymes that are involved in the degradation of the ECM, i.e., metalloproteinases (MMPs) and their inhibitors, tissue inhibitor of metalloproteinase (TIMP). ${ }^{15,16}$ Since both MMP and TIMP maintain the integrity of the ECM and cellular basal membrane, an imbalance between MMP and TIMP activity can lead to elevated degradation of ECM with loss of connective tissue of the lamina propria, breakdown of the vascular basal membrane that facilitates vessel permeability and the consequent subepithelial oedema and formation of polyps. ${ }^{12,17}$ The tissue remodelling during CRSwNP is also associated with the Epithelialto-Mesenchymal Transition, a cellular process in which epithelial cells can have an impact on the development and progression of ECM alteration comparable to that of fibroblasts. ${ }^{18}$ In EMT, epithelial cells show the loss of cell-cell adhesion and polarity and slowly gain myofibroblast markers as they lose their epithelial status. These cells then leave the epithelial layer (mainly due to the E-cadherin loss) and accumulate in the interstitium, thus beginning the ECM synthesis. ${ }^{19}$

Growing interest has been shown in advanced glycosylation end products (AGEs), the products of non-enzymatic glycation and oxidation of proteins and lipids, since their interaction with receptor of advanced glycosylation end products (RAGE) and other receptors activate several pathways involved in many human diseases characterised by a deregulation of collagen metabolism. ${ }^{20-23}$ Recent in vitro studies demonstrated that the interaction between AGE and RAGE seem capable of inducing connective remodelling through MMP-1, TIMP and changes in $\mathrm{p} 38$ mitogen-activated protein chinasi (MAPK) and NF-kB. ${ }^{24}$ During recurrent rhinosinusitis, RAGE is overexpressed in the epithelial cells of the sinonasal mucosa obtained from patients affected by CRSwNP ${ }^{25}$ and in the same patients, MAPK/ extracellular signal-regulated kinases (ERK) is activated showing that this pathway is also involved in the inflammatory process and in the pathogenesis of CRSwNP. ${ }^{26}$

Since a complex network of processes including epithelial damage, inflammatory infiltration, EMT and tissue remodelling occur in CRSwNP and the underlying molecular mechanisms of these events have not been completely elucidated, the aim of this study was to investigate the interaction between the AGEs/RAGE/ERK signalling pathway and TGF $\beta /$ Smads in patients affected by CRSwNP.

\section{Patients and Methods}

\section{Patients selection}

This study was carried out (March 2018-March 2019) by selecting 30 patients divided into two groups. The control group consisted of 16 patients (eight males and eight females) undergoing septoplasty (STPL) for nasal stenosis and endoscopic sinus surgery for chronic sinusitis. The case group was comprised of 14 patients (twelve males and two females) suffering from CRSwNP undergoing endoscopic surgery. Patient selection was done according to different criteria. In particular, people less than 18 years of age, patients with diagnoses of single and unilateral NP and patients treated with antiplatelet and/or anticoagulant drugs were excluded.

The preoperative clinical history of all patients was careful evaluated revealing the presence of recurrent CRSwNP-correlated risk factors such as allergies, smoking and employment-related factors.

The Institutional Ethic Committee (n. 9993) approved the investigation protocol and all eligible patients signed a consent form regarding the processing of personal data, allowing the excision of tissue and its use for this study.

\section{Tissue collection and preparation}

Biopsies were cleaned and immediately put in $4 \%$ buffered formalin for $3 \mathrm{~h}$ at room temperature. Thereafter, fragments were embedded in low temperature fusion paraffin for histological and immunohistochemistry evaluation. A fraction of the same tissue was stored at $-80^{\circ} \mathrm{C}$ for Western blot analysis. 


\section{Microscopic evaluation of nasal polyps}

Serial 3- $\mu \mathrm{m}$-thick sections were stained using Haematoxylin and Eosin (H\&E), to assess the general tissue morphology, Masson's Trichrome and Periodic Acid-Schiff reaction (PAS) to evaluate the deposition of connective tissue and to identify glandular and epithelial glycoprotein compound, respectively. The stained sections were then observed under an Olympus BX51 light microscope (Olympus Optical Co. Ltd., Tokyo, Japan).

\section{Immunohistochemistry analysis}

Biopsies were cleaned and immediately put in $4 \%$ buffered formalin for $3 \mathrm{~h}$ at room temperature and embedded in low-melting paraffin. Serial sections of $3 \mu \mathrm{m}$ in thickness were incubated in methanol and 3\% hydrogen peroxidase solution for $40 \mathrm{~min}$ and then rinsed in phosphate buffered saline (PBS). Specimens were incubated overnight at $4^{\circ} \mathrm{C}$ with the following antibodies:

AGE (ab23722: Abcam, Cambridge, UK; dilution 1:500); RAGE (pA1-075: Thermo Fisher Scientific Inc., Waltham, MA, USA; dilution 1:100); p-ERK (sc-7383; Santa Cruz Biotechnology, Santa Cruz, CA, USA; dilution 1:200); MMP-3 (sc-6839; Santa Cruz Biotechnology; dilution 1:50); TGF- $\beta 1$ (sc8784; Santa Cruz Biotechnology; dilution 1:200); Smad2/3 (sc6202; Santa Cruz Biotechnology; dilution 1:300); Collagen I (sc8784; Santa Cruz Biotechnology; dilution 1:200); Collagen III (sc8781; Santa Cruz Biotechnology; dilution 1:200); $\alpha$ - SMA (sc32251: Santa Cruz Biotechnology; dilution 1:200; E-cadherin (sc7870: Santa Cruz Biotechnology; dilution 1:100); Vimentin (sc6260: Santa Cruz Biotechnology; dilution 1:100); IL-6 (sc-28343: Santa Cruz Biotechnology; dilution 1:100).

Fragments were then rinsed with PBS for $10 \mathrm{~min}$ and incubated with a labelled streptavidin-biotin-peroxidase conjugate kit (Dako Envision HRP: K 5007, Dako A/S, Glostrup, Denmark) for $20 \mathrm{~min}$. After rinsing in PBS for $10 \mathrm{~min}$, the sections were incubated with 3,3-diaminobenzidine-tetrahydrochloride (DAB: K3468, Dako Cytomation, North America Inc., Carpinteria, CA, USA) for 1-3 min. Lastly, the samples were counterstained with Mayer's Haematoxylin and observed under a photomicroscope (Olympus BX51 light microscope; Olympus Optical Co. Ltd).

To control the immunoreaction specificity, negative and positive controls were performed for all reactions. For negative controls, sections were treated omitting the primary antibody and replacing it with $0.01 \mathrm{M}$ PBS.

All sections were evaluated independently by two experienced pathologists and examined under an Olympus BX51 light microscope (Olympus Optical Co. Ltd.).

\section{Semi-quantitative immunohistochemistry evaluation and statistical analysis}

Quantitative comparison of immunohistochemical staining was performed by Image J, a digital image analysis public domain software (U.S. National Institutes of Health, Bethesda, MD, available at: https://imagej.nih.gov/ij/) for all antibodies tested in the study. The IHC images employed were stained with DAB and Haematoxylin and five microscopic fields were selected from each samples of control and CRSwNP, photographed at the same magnification (Original Magnification 10×, Scale bar: $100 \mu \mathrm{m}$ ), saved as high-resolution images and then analysed with an IHC profiler, a specific Image J plugin. The microphotographs underwent spectral deconvolution method of DAB/Haematoxylin. For each of the photographs, the software analysed the pixel intensity splitting it in threshold values: negative, low positive, positive, and high positive. Taking this into account, we obtained the whole immunopositivity by summing the three different grades of positivity. The immunopositivity was expressed as a percentage of the total software-classified areas and the data obtained were plotted on his- tograms. Results were expressed as means $\pm \mathrm{SD}$ and considered statistically significant at a $\mathrm{P}$-value $<0.05$.

\section{Western blot analysis}

The evaluation of marker levels was performed on proteins extracted by homogenisation of patients' tissues in lysis buffer $2 \mathrm{x}$ (Cell Signalling Technology-9802, Danvers, MA, USA) added with proteases and phosphatases inhibitors (Cell Signalling Technology-9803). The amount of proteins for each sample was calculated with a Bradford assay, and electrophoresis was run in denaturant conditions. The protein was transferred on a nitrocellulose membrane and incubated with the following primary antibodies: AGE (ab 23722: Abcam), RAGE (pA1-075: Thermo Fisher Scientific Inc., Waltham, MA, USA), ERK 1/2 (44-6544: Thermo Fisher, Scientific Inc.), p-ERK (sc-7383: Santa Cruz Biotechnology) and MMP3 (sc-6839: Santa Cruz Biotechnology) at concentrations suggested by the datasheets. Finally, the identification of the specific chemiluminescent bands was performed by exposure of the nitrocellulose membrane, previously incubated with the corresponding secondary antibodies, to ECL (Chemiluminescent Substrate Pico. COD. EMP001005 Euroclone, Milan, Italy).

\section{Results}

\section{Whole mount histology and immunohistochemistry}

Histomorphological evaluation with H\&E (Figure 1 A,B), Masson's Trichrome (Figure $1 \mathrm{C} / \mathrm{C} 1, \mathrm{D} / \mathrm{D} 1$ ) and histochemical staining using PAS (Figure 2 A/A1, B/B1) allowed demonstration of the substantial differences in the general aspects of glands, inflammatory infiltration and ECM between samples from controls and patients with NP. In particular, the nasal mucosa of control samples was morphologically normal (Figure 1A) and smooth, with ciliated columnar epithelial cells separated from the thick lamina propria by a thin layer of basement membrane.

In the submucosa of the control samples several seromucous glands were immersed in a connective tissue with elastic fibres (Figure $1 \mathrm{C} / \mathrm{C} 1$ ).

In the surface epithelium of CRSwNP samples we observed an accumulation of glycoproteins in the cytoplasm of goblet cells, with a thickened of subepithelial connective tissue and oedema (Figure $2 \mathrm{~B} / \mathrm{B} 1$ ). Moreover, in the same group, a remodelling of the submucosal layer, characterised by loose connective tissue with inflammatory infiltration, a reduction of number with a poor PAS positivity of glands showing dysmorphisms, winding appearance and luminal dilations were present (Figure 1B, D/D1 and Figure 2 $\mathrm{B} / \mathrm{B} 1)$

Immunohistochemistry for AGEs showed a similar low immunolabeling in the epithelium both in fragments from polyps and in controls (Figure 3 A,B). On the contrary, we observed a marked overexpression of the same antigen in the subepithelium and in the connective tissue of the lamina propria and submucosa in fragments from pathological samples (Figure 3B) respect to controls (Figure 3A). As far as RAGE expression, it was generically overexpressed in polyps (Figure 3E) in contrast to controls (Figure 3D); in particular, the immunopositivity was evident in the deeper region of the lamina propria, in the submucosa and around the vessels (Figure 3E). These data were confirmed by semi-quantitative analysis (Figure $3 \mathrm{C}, \mathrm{F}$ ).

Immunohistochemistry for p-ERK (Figure $3 \mathrm{G}, \mathrm{H}$ ) revealed a moderate positivity in the connective tissue of the tunica mucosa and a lower immunoreaction in the surface epithelium and glands of the control samples (Figure $3 \mathrm{G}$ ). On the contrary, a relevant 
enhancement of the immunopositivity for the same MAPK, both in the lamina propria and the stroma that encircled the submucosal glands of the NP nasal polyps (Figure $3 \mathrm{H}$ ), was reported. The increased levels of p-ERK in polyps' fragments respect to controls was also highlighted by semi-quantitative evaluation (Figure 3I).

An increase of immunostaining for MMP3 was observed in the lamina propria and the submucosa of the pathological tissue (Figure 3M) when compared to normal samples (Figure 3L). This moderate difference in immunolabeling between the two groups was confirmed by semi-quantitative analysis $(60 \%$ in NP vs $40 \%$ in controls) (Figure 3N). When comparing TIMP1 expression between the two groups, we identified a different topographic distribution of immunoreaction as TIMP1 was located in the subep- ithelium in controls (Figure 3O), while in NP, the immunostaining labelled the deeper region of the submucosa rather than the lamina propria (Figure 3P). Morphological data were confirmed by semiquantitative analysis, which showed no differences in TIMP1 labelling (Figure 3Q).

Immunohistochemistry and semi-quantitative analysis for the TGF- $\beta /$ Smads pathway did not show any significant differences in polyps and controls regarding the expression of TGF- $\beta 1$, Smad23 , Collagen I-III and $\alpha$-SMA (Figure 4), demonstrating that this cascade seems not to be involved in ECM remodelling and does not represent an alternative cascade to AGEs/RAGE (Figure 4).

To demonstrate the ability of AGE/ERK signalling to induce the EMT process, we tested E-cadherin (epithelial) and Vimentin
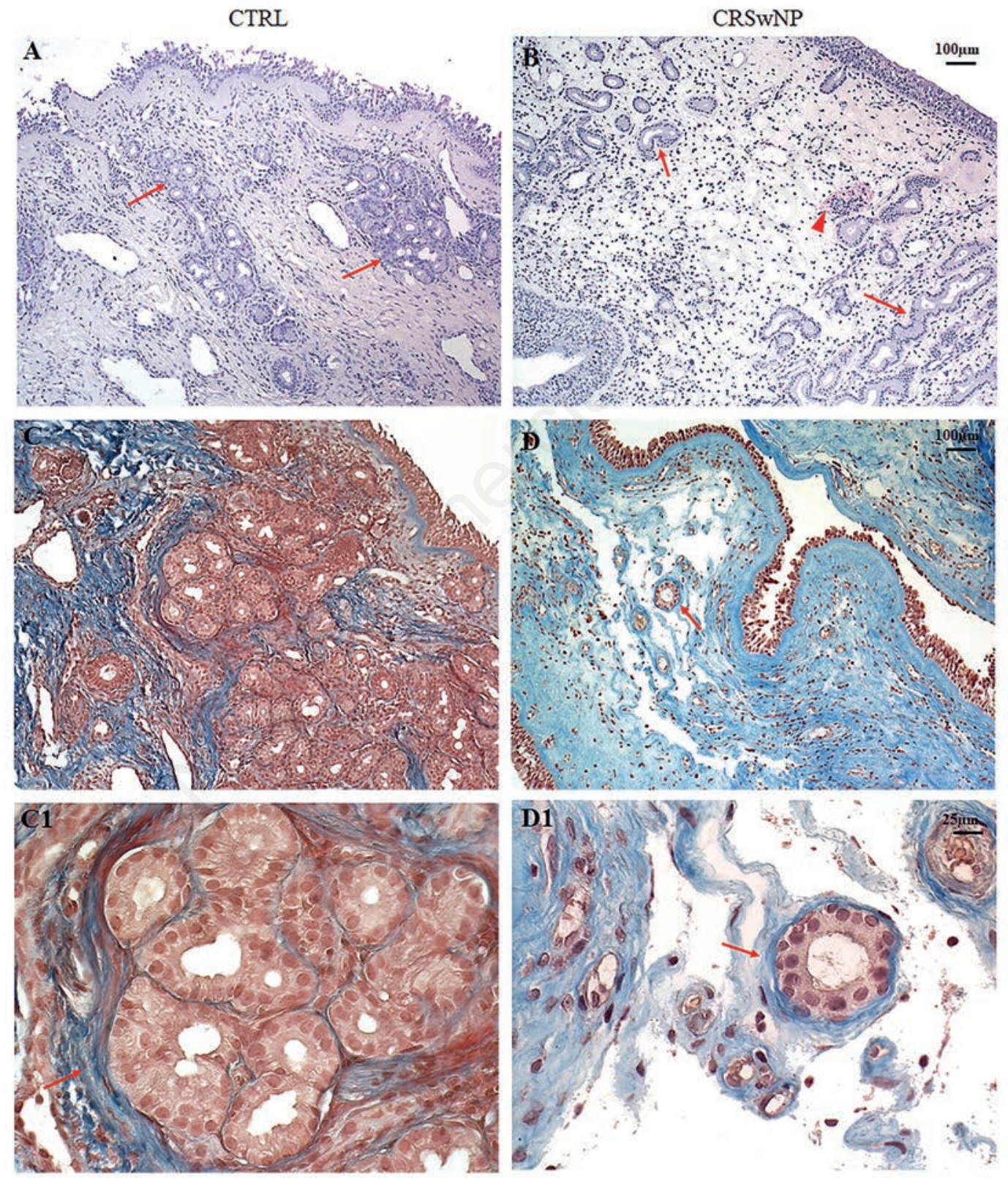

Figure 1. H\&E and Masson's Trichrome staining. In control (CTRL) samples (A, C/C1) the microphotograph showed a normal morphology of nasal mucosa with several dense glandular aggregates encircled by a dense network of elastic fibres (arrows) respect to CRSwNP samples (B, D/D1) where glands appeared dysmorphic, isolated and partly surrounded by thin fibres of connective tissue (arrows). Inflammatory infiltrate was also detected in the CRSwNP group (B-head arrow). Original magnification 10x, scale bar: $100 \mu \mathrm{m}$. 
and IL-6 (mesenchymal) markers (Figure 5). As expected, E-cadherin immunopositivity was higher in controls (Figure $5 \mathrm{~A} / \mathrm{A} 1$ ) than in polyps (Figure $5 \mathrm{~B} / \mathrm{B} 1$ ), as confirmed by semi-quantitative analysis (Figure 5C). On the contrary, IL-6 and Vimentin expression was mild in the control group (Figure $5 \mathrm{D}, \mathrm{G}$ ) and increased significantly in patients affected by NP (Figure $5 \mathrm{E}, \mathrm{H}$ ), confirming the switch from epithelial to mesenchymal phenotype. These data were confirmed by semi-quantitative analysis (Figure 5 F,I)

In accordance with the immunohistochemistry results, Western blot analysis revealed high levels of AGE, RAGE and MMP3 in polyps samples compared to controls (Figure 6). As far as ERK level we did not observe significant differences between the two groups, while we noticed an increased level of p-ERK in NP confirming the activation of ERK signalling in patients with CRSwNP (Figure 6). These data confirmed the involvement of the AGE/RAGE cascade in ECM remodelling and in the EMT process occurring in NP disease.

\section{Discussion}

The morphological aspects of NP have been well described from the histological point of view but the cellular mechanisms underlying the pathogenesis of these events is not fully understood.

Previous studies have indicated that several molecular pathways are involved in the pathogenesis of NP and most of these studies primarily focused on single different pathways involved in Chronic rhinosinusitis (CRS), concentrating on detecting the proteins or gene expression in the context of a specific cascade. As the correlation among the several cascades still remains unclear, our study evaluated, in controls vs CRSwNP patients, the immunohistochemical alterations of nasal mucosa induced by AGE/RAGE in an independent or parallel manner to the TGF $\beta /$ Smads pathway, as well as AGE/RAGE influence the remodelling of mucosa in NP. Tissue remodelling is orchestrated by the activation of a profibrotic programme in which lots of mediators can induce fibroblasts to
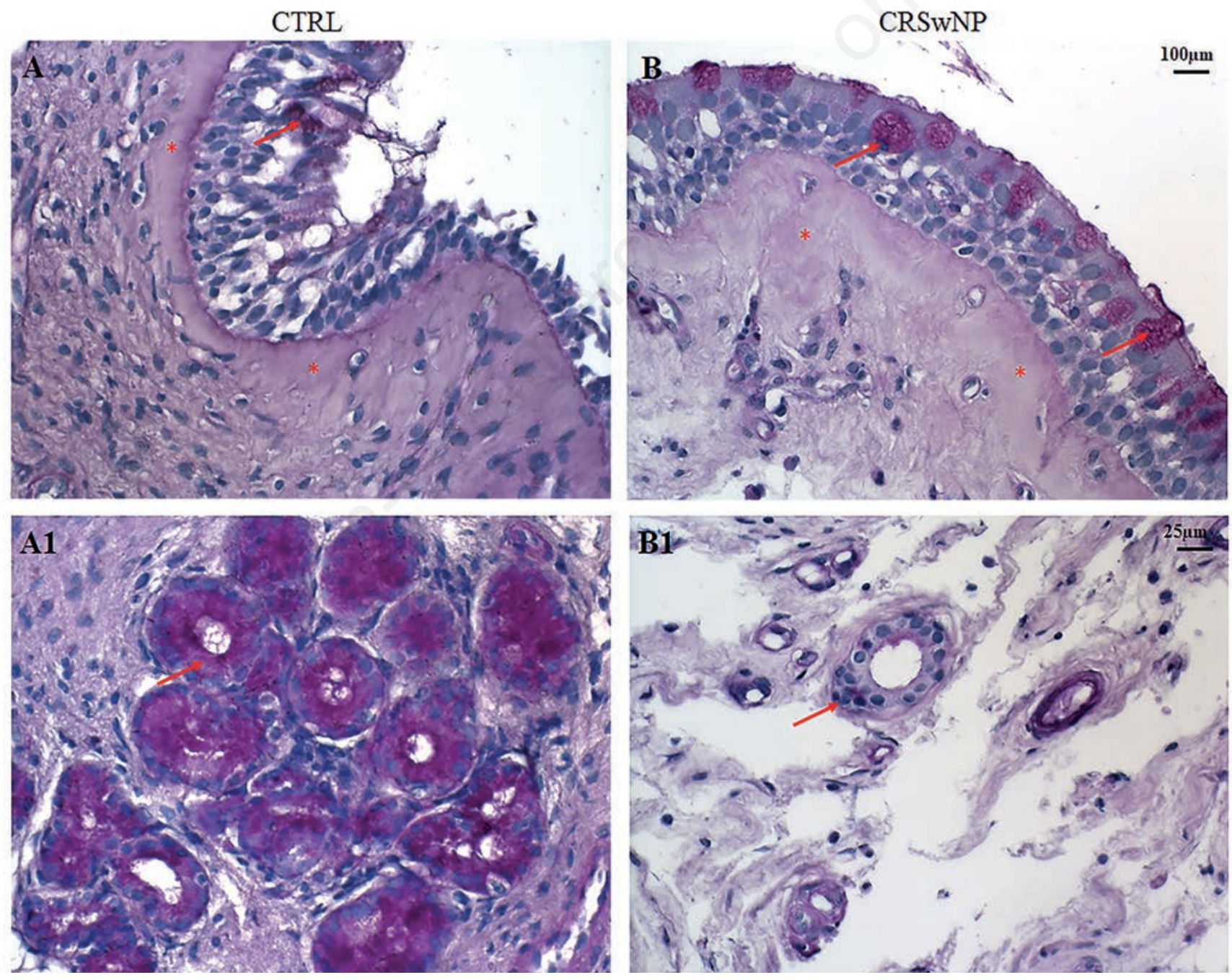

Figure 2. PAS staining. In the epithelium of CTRL samples (A) a poor accumulation of glycoproteins in the cytoplasm of few goblet cells was found (arrow), while a marked PAS positivity in glands was detected (A1, arrow). On the contrary, in the epithelium of CRSwNP samples (B) an increase of glycoprotein accumulation was found in the goblet cells (arrows) while glands were PAS negative (B1, arrow). A thickened of subepithelial connective tissue was observed in CRSwNP compared to CTRL (asterisks). (A, B) Original magnification 10x, scale bar: $100 \mu \mathrm{m}$; (A1, B1) Original magnification 40x, scale bar: $25 \mu \mathrm{m}$. 

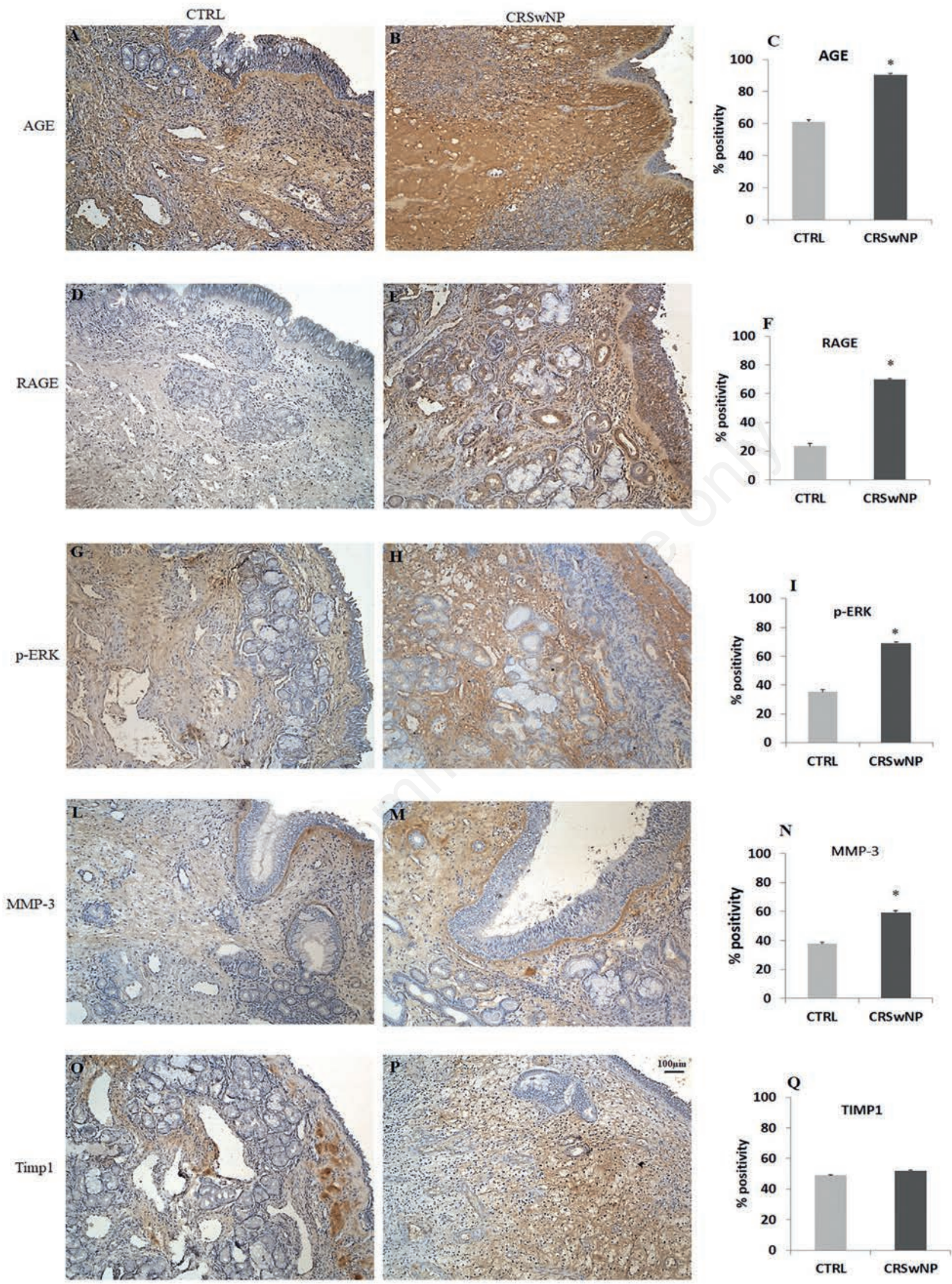

Figure 3. Immunohistochemistry and semi-quantitative analysis for AGE, RAGE, p-ERK, MMP3 and TIMP1. In CTRL samples (A,D,G,L) all markers tested were mildly expressed in contrast to the CRSwNP samples $(\mathrm{B}, \mathrm{E}, \mathrm{H}, \mathrm{M})$, which showed a significant increase of immunopositivity. Regarding TIMP1, no quantitative differences were detected between the two groups $(\mathrm{O}, \mathrm{P})$. Data were confirmed by semi-quantitative analysis (C,F,I,N,Q). Original magnification 10x, scale bar: $100 \mu \mathrm{m}$. 
collagen deposition other than by a morphological or functional defect in the epithelial barrier due to EMT, another event thought to be one of the pathophysiological processes involved in CRS. ${ }^{27,28}$

TGF- $\beta$ is considered a master switch in tissue remodelling and degradation of the basal membrane and it has been shown to be closely associated with chronic respiratory diseases including CRS. Clear differences have been demonstrated in the TGF- $\beta$ signalling pathway between CRSwNP and CRSsNP and this supports the hypothesis that the TGF- $\beta$ pathway plays a different role in the induction of fibrosis or its dysregulation in the nasal mucosa. We
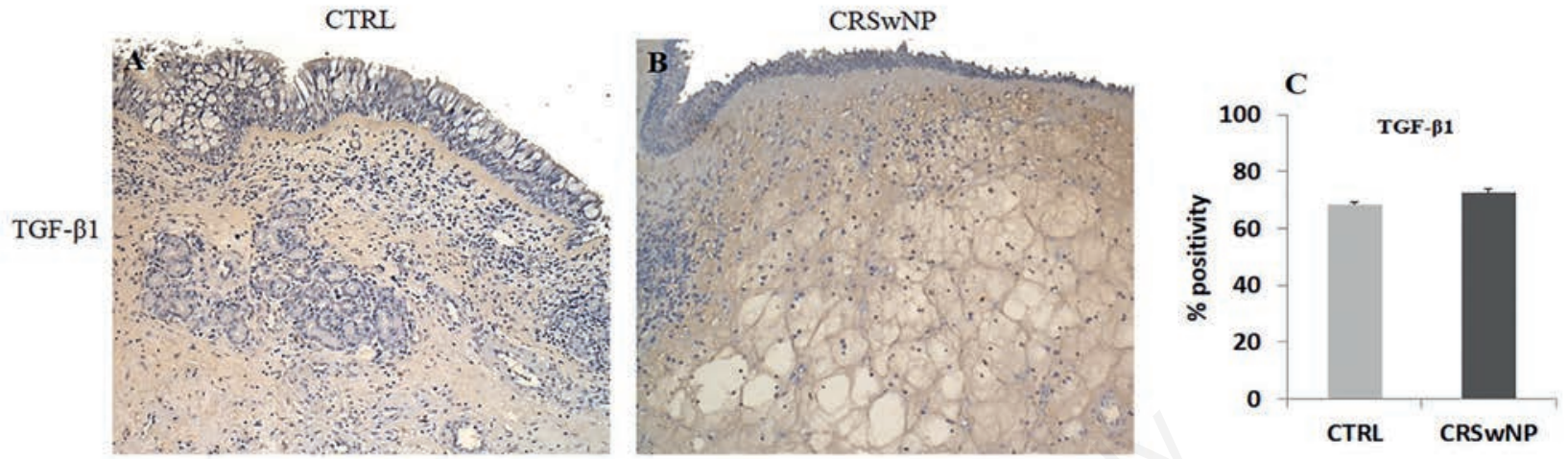

Smad $2 / 3$
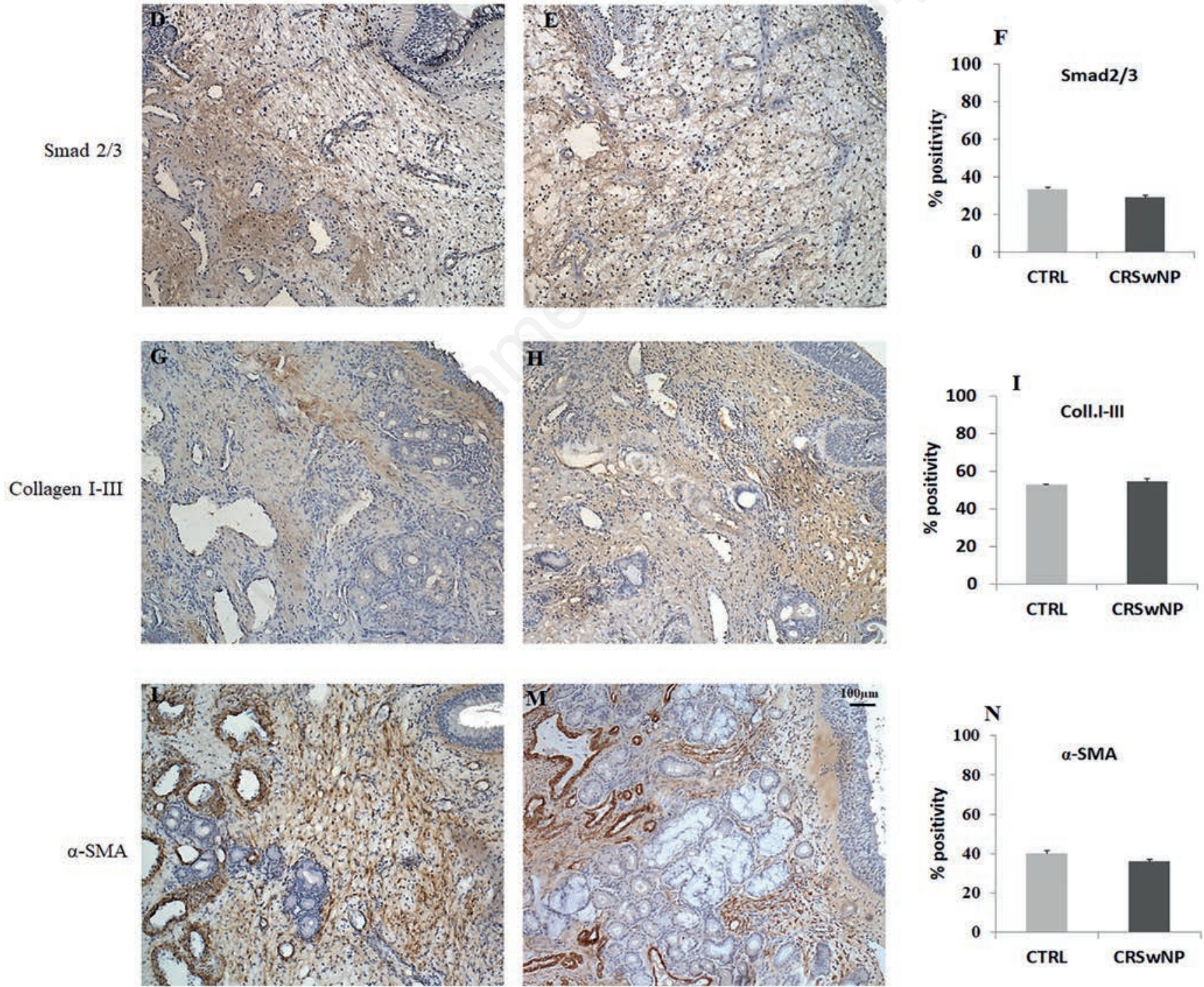

Figure 4. Immunohistochemistry and semi-quantitative analysis for TGF- $\beta 1$, Smad2/3, Collagen I-III and $\alpha$-SMA. No significative differences in immunopositivity between CTRL $(A, D, G, L)$ and CRSwNP $(B, E, H, M)$ samples were found as confirmed by semi-quantitative evaluation $(\mathrm{C}, \mathrm{F}, \mathrm{I}, \mathrm{N})$. Original magnification $10 \times$, scale bar: $100 \mu \mathrm{m}$. 
found that TGF- $\beta 1$ and Smad2/3 proteins' immunopositivity were present in nasal mucosa from both CRSwNP and CTRL patients with no quantitative differences. Therefore, it is conceivable that the TGF- $\beta /$ Smads pathway is not essential to polyps' formation and other signalling pathways may contribute to the disease.
Growing interest has been shown in AGEs, the products of non-enzymatic glycation and oxidation of proteins and lipids, since their interaction with RAGE and other receptors activate several pathways including ERK and MAPK, which are involved in many human diseases characterised by a tissue remodelling. There is evi-
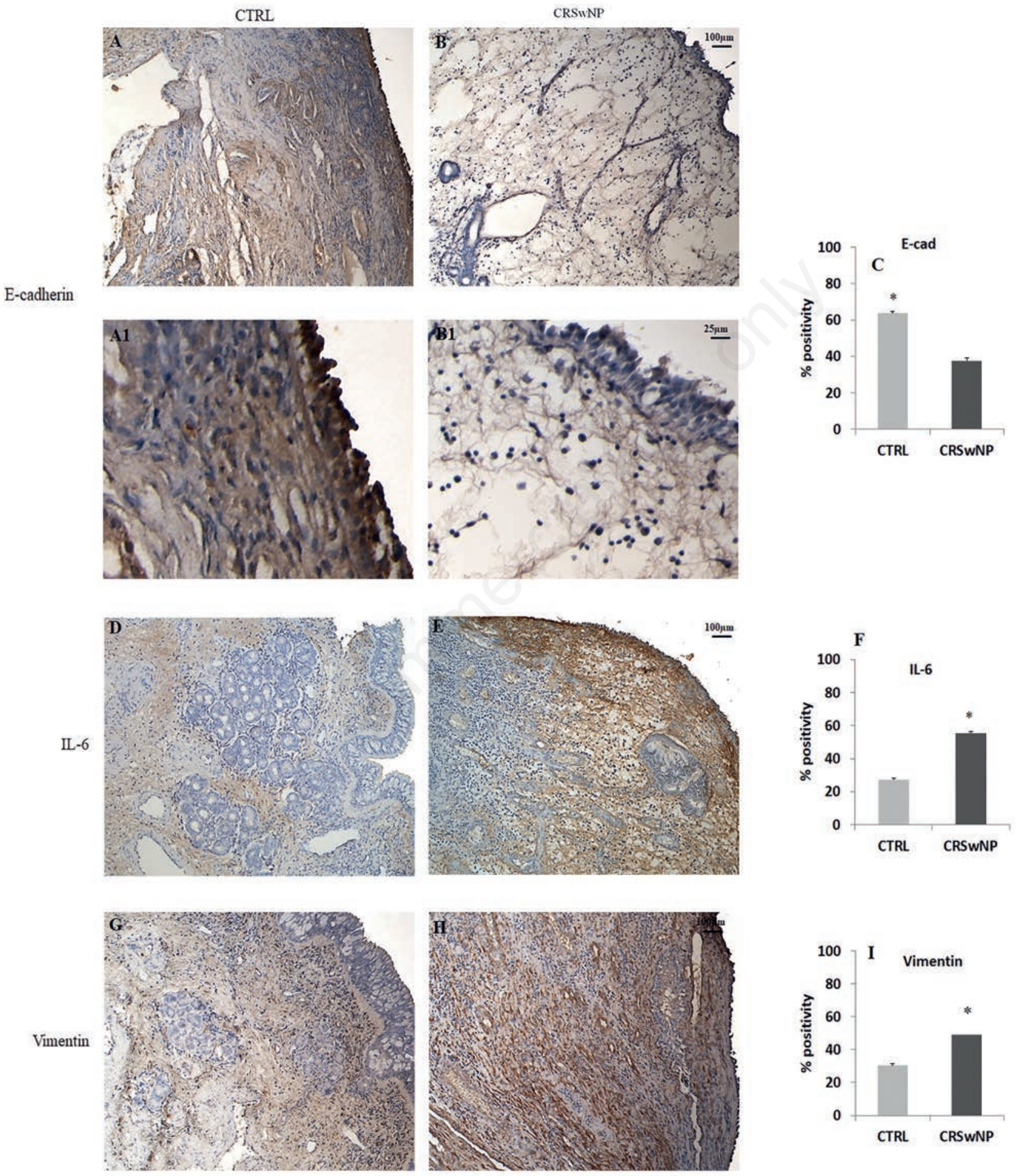

Figure 5. Immunohistochemistry and semi-quantitative analysis for E-cadherin, IL-6 and Vimentin. In CTRL samples (A) a marked epithelial positivity for E-cadherin was found in contrast to CRSwNP, where the expression was mild (B). On the contrary, both IL-6 and Vimentin were expressed to a greater extent in CRSwNP fragments $(\mathrm{E}, \mathrm{H})$ compared to CTRL $(\mathrm{D}, \mathrm{G})$. These data were confirmed by semi-quantitative analysis (C,F,I). Original magnification 10x, scale bar: $100 \mu \mathrm{m}$. 
dence that the ERK pathway, numbered in the MAPKs family, is activated in CRSwNP and represents a critical intracellular mediator implicated in the pathogenesis of CRSwNP. ${ }^{26}$

ERK can mediate the increase of metalloproteinases levels in a TGF $\beta /$ Smads independent manner; therefore, it is conceivable that the increased levels of p-ERK and MMP3 in the nasal mucosa of CRSwNP could be due to the activation of the AGE/RAGE pathway and TGF- $\beta /$ Smad signalling seems to play a marginal role in the development of CRSwNP. In confirmation of these findings, we observed that collagen I/III and $\alpha$ SMA expressions showed no significant differences between the two group of samples, demonstrating that, in NP, a tissue remodelling process involving fibroblasts or their transdifferentiation into myofibroblasts with enhanced collagen synthesis does not occur.

MMPs and their inhibitors TIMPs are considered to play an additive role in NP formation. MMP3 was expressed in the lamina propria and submucosa in CRSwNP, appearing responsible for microvascular permeability leading to oedema and ECM protrusion. Simultaneously, there was no TIMP1 suppression and its low immunolabeling was similar in the two groups of patients, suggesting that the imbalance between high MMPs and a limited expression of their inhibitors TIMPs cannot prevent MMPs from overactivation. This event is capable of aggravating the oedema of the nasal mucosa through breakdown of the vascular basal membrane and increase of vessel permeability.

Recently, EMT has been observed in CRS and NP tissues and is thought to contribute to the pathophysiological process in CRSwNP. ${ }^{18}$ During EMT, the epithelial cells lose their typical morphology, apicobasal polarity and cell to cell junctional attachments and acquire mesenchymal properties causing a mucosal barrier disruption and pseudocyst formation. In particular, in patients with CRSwNP, epithelial cells undergoing EMT show a loss of epithelial markers such as E-cadherin and a gain of mesenchymal markers such as $\alpha$-SMA, Vimentin and Snail. ${ }^{29,30}$ Since in our study the p-ERK level was correlated with markers of EMT in NP such as IL-6 and Vimentin, we speculate that ERK signalling influenced by the AGE/RAGE pathway could induce EMT, causing an epithelial disruption with a decrease of epithelial cohesion and integrity together with a transdifferentiation of epithelial cells into mesenchymal cells contributing to stromal tissue oedema, tissue

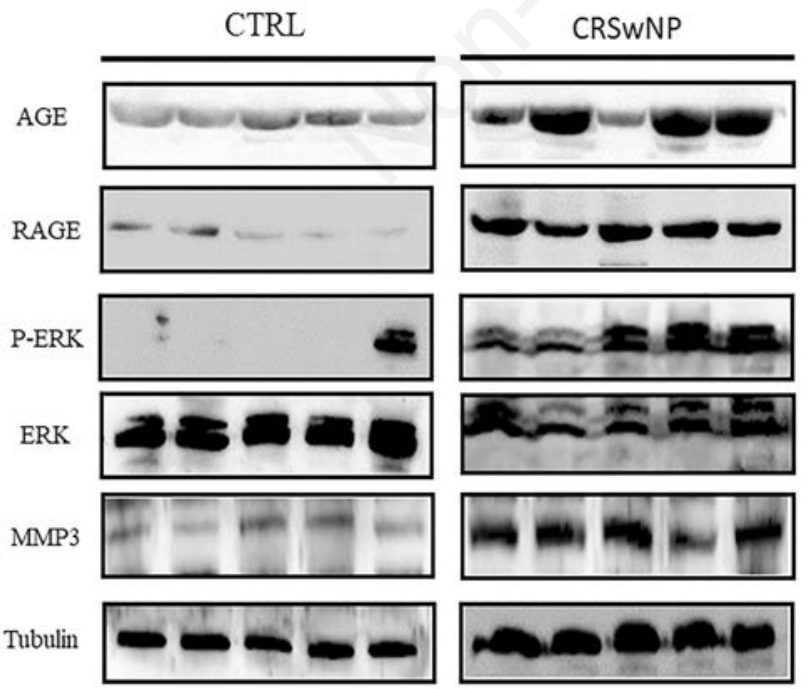

Figure 6. Western blot evaluation of AGE, RAGE, p-ERK, ERK and MMP3. Increased levels of all markers were found in CRSwNP in contrast to CTRL samples. remodelling and pseudocyst formation.

CRS is a complex inflammatory disease and many factors contribute to its development. Our findings, presented here, suggest that the activation of the AGE/RAGE/ERK rather than TGF $\beta /$ Smads pathway might deserve a leading position in sinus mucosa hyperproliferation and recurrent NP formation. Our results also suggest that EMT, with the protrusion of connective tissue through an epithelial barrier defect and the subsequent re-epithelisation, could also be correlated to the overexpression of AGE/RAGE/ERK driven by epithelial-derived inflammatory cytokines.

Our preliminary data confirm the extremely complex pathogenesis of CRSwNP. However, further studies are required to validate our hypothesis that the AGE/RAGE/ERK pathway and EMT might work together in promoting tissue remodelling in NP during CRS and it is reasonable to speculate that the pharmacological control of this intracellular signalling could represent a promising strategy in the future.

\section{References}

1. Fokkens WJ, Lund VJ, Mullol J, Bachert C, Alobid I, Baroody F, et al. EPOS European Position Paper on rhinosinusitis and nasal polyps. A summary for otorhinolaryngologists. Rhinology 2012;50:1-12.

2. Schleimer RP. Immunopathogenesis of chronic rhinosinusitis and nasal polyposis. Annu Rev Pathol 2017;12:331-57.

3. Sin A, Terzioglu E, Kokuludag A, Veral A, Sebik F, Karci B, et al. Allergy as an etiologic factor in nasal polyposis. J Investig Allergol Clin.Immunol 1997;7:234-7.

4. Collins MM, Pang YT, Loughran S, Wilson JA. Environmental risk factors and gender in nasal polyposis. Clin Otolaryngol Allied Sci 2002; 27:314-7.

5. Gorgulu O, Ozdemir S, Canbolat EP, Sayar C, Olgun MK, Akbaş Y. Analysis of the roles of smoking and allergy in nasal polyposis. Ann Otol Rhinol Laryngol 2012;121:615-9.

6. Li X, Tao Y, Li X. Expression of MMP-9/TIMP-2 in nasal polyps and its functional implications. Int J Clin Exp Pathol 2015;8:14556-61.

7. Severi C, Sferra R, Scirocco A, Vetuschi A, Pallotta N, Pronio A, et al. Contribution of intestinal smooth muscle to Crohn's disease fibrogenesis. Eur J Histochem 2014;58:2457.

8. Vetuschi A, D'Alfonso A, Sferra R, Zanelli D, Pompili S, Patacchiola $\mathrm{F}$, et al. Changes in muscularis propria of anterior vaginal wall in women with pelvic organ prolapse. Eur J Histochem 2016;60:2604.

9. Sferra R, Pompili S, Festuccia C, Marampon F, Gravina LG, Ventura L, et al. The possible prognostic role of histone deacetylase and transforming growth factor $\beta /$ Smad signaling in high grade gliomas treated by radio-chemotherapy: A preliminary immunohistochemical study. Eur J Histochem 2017; 61:2732.

10. Smith RS, Smith TJ, Blienden TM, Phipps RP. Fibroblasts as sentinel cells-synthesis of chemokines and regulation of inflammation. Am J Pathol 1997;151:317-22.

11. Teran LM, Mochizuki M, Bartels F, Valencia EL, Nakajima T, Hirai K, Schröder JM. Th1 and Th2 type cytokines regulate the expression and production of eotaxin and RANTES by human lung fibroblasts. Am J Respir Cell Mol Biol 1999;20:777-86.

12. Cho JS, Kang JH, Um JY, Han IH, Park IH, Lee HM. Lipopolysaccharide induces proinflammatory cytokines and MMP production via TLR4 in nasal polyp derived fibroblast and organ culture. PLoS One 2014;9:e90683.

13. Van Bruaene N, Derycke L, Perez-Novo CA, Gevaert P, 
Holtappels G, De Ruyck N, et al. TGF $\beta$ signaling and collagen deposition in chronic rhinosinusitis. J Allergy Clin Immunol 2009;124:253-9. doi:10.1016/j.jaci.2009.04.013.

14. Kou W, Hu GU, Yao HB, Wang X, Shen Y, Kang HY, et al. Regulation of Transforming Growth Factor $\beta 1$ activation and expression in the tissue remodeling involved in chronic rhinosinusitis. ORL J Otorhinolaryngol Relat Spec 2012;74:172-8.

15. Hwang KE, Shon YJ, Cha BK, Park MJ, Chu MS, Kim YJ, et al. Tissue inhibitor of metalloproteinase1 is responsible for residual pleural thickening in pleural tuberculosis. Tohoku $\mathrm{J}$ Exp Med 2015;235:327-33.

16. Zang C, Chen L, Gu Y. Polymorphisms of MMP-1 and MMP3 and susceptibility to rheumatoid arthritis: a meta-analyses. $\mathrm{Z}$ Rheumatol 2015;74:258-62.

17. Katainen E, Kostamo K, Virkkula P, Sorsa T, Tervahartiala T, Haapaniemi A, et al. Local and systemic proteolytic responses in chronic rhinosinusitis with nasal polyposis and asthma. Int Forum Allergy Rhinol 2015;:294-302.

18. Konnecke M, Burmeister M, Pries R, Böscke R, Bruchhage KL, Ungefroren H, et al. Epithelial Mesenchymal Transition in chronic rhinosinusitis: differences revealed between epithelial cells from nasal polyps and inferior turbinates. Arch Immunol Ther Exp (Warsz) 2017;65:157-73. doi:10.1007/s00005-0160409-7.

19. Di Gregorio J, Sferra R, Speca S, Vetuschi A, Dubuquoy C, Desreumaux P, et al. Role of glycogen-synthase kinase- $3 \beta$ and PPAR- $\gamma$ on Epithelial-to-Mesenchymal Transition in DSSinduced colorectal fibrosis. PLoS One 2017;12:e0171093. doi:10.1371/journal.pone.0171093.

20. Lohwasser C, Neureiter D, Weigle B, Kirchner T, Schuppan D. The receptor for advanced glycation end products is highly expressed in the skin and upregulate by advanced glycation end products and tumor Necrosis Factor-Alpha. J Invest Dermatol 2006;126:291-9. doi:10.1038/sj.jid.5700070.

21. Ramasamy R, Yan SF, Schmidt AM. Advanced glycation end products: from precursor to RAGE: round and round we go. Amino Acids 2012;42:1151-61.
22. Yamagishi S, Fukami K, Maatsui T. Evaluation of tissue accumulation levels of advances glycation end products by skin autofluorescence: a novel marker of vascular complications in high risk patients for cardiovascular disease. Int $\mathrm{J}$ Cardiol 2015;185:263-8.

23. Hu H, Jiang H, Ren H, Hu X, Wang X, Han C. AGEs and chronic subclinical inflammation in diabetes: disorders of immune system. Diabetes Metab Res Rev 2015;31:127-37.

24. Chen Y, Wang X, Feng W, Hua KQ. Advanced glycation end products decrease collagen I levels in fibroblasts from the vaginal wall of patients with POP via the RAGE, MAPK and NF-kB pathways. Int J Molec Med 2017;40:987-98 doi:10. 3892/ijmm.2017.3097.

25. Dzaman K, Zagor M, Molinska-Glura M, Krzeski A. High motility group box1 (HMGB1) protein and its receptor for advanced glycation end products (RAGE) expression in chronic rhinosinusitis without nasal polyps. Folia Histochem Cytobiol 2015;53:70-8. doi:10.5603/FHC.a2015.0007.

26. Linke R, Pries R, Konnecke M, Bruchhage KL, Böscke R, Gebhard M, et al. The MEK1/2-ERK1/2 pathway is activated in chronic rhinosinusitis with nasal polyps. Arch Immunol Ther Exp (Warsz) 2014;62:217-29. doi:10.1007/s00005-0140281-2.

27. Esplugues E, Huber S, Gagliani N, Hauser AE, Town T, Wan YY, et al. Control of TH17 cells occurs in the small intestine. Nature 2011;475:514-8.

28. Zang N, Van CK, Gevaert E, Bacher C. Barrier function of the nasal mucosa in health and type-2 biased airway diseases. Allergy 2016;71:295-307.

29. Kouro T, Takatsu K. IL-5 and eosinophil-mediated inflammation: from discovery to therapy. Int Immunol 2009;21:1303-9.

30. Zhou J, Zhang C, Pan J, Chen L, Qi ST. Interleukin-6 induces an epithelial to mesenchymal transition phenotype in human adamantinomatous craniopharyngioma cells and promotes tumor cell migration. Mol Med Rep 2017;15:4123-31.

Received for publication: 21 October 2019. Accepted for publication: 14 January 2020.

This work is licensed under a Creative Commons Attribution-NonCommercial 4.0 International License (CC BY-NC 4.0).

(C) Copyright: the Author(s), 2020

Licensee PAGEPress, Italy

European Journal of Histochemistry 2020; 64:3079

doi:10.4081/ejh.2020.3079 